Table 1. MCII and PASS of pain and function instruments in hand OA patients in two settings.

\begin{tabular}{|c|c|c|c|c|c|}
\hline \multirow[b]{2}{*}{ Instrument } & \multicolumn{3}{|c|}{ MCII $(95 \% \mathrm{Cl})$ in RCT† } & \multicolumn{2}{|l|}{ PASS $(95 \% \mathrm{Cl})$} \\
\hline & Absolute units & Percentage & {$[\mathrm{n}]$} & $\mathrm{RCT}(\mathrm{n}=68)$ & Cohort $(n=126)$ \\
\hline \multicolumn{6}{|l|}{$\mathrm{MHQ}$} \\
\hline Overall function, $0-100^{*}$ & $3.4(-2.7 ; 9.5)$ & $3.6(-9.1 ; 16.3)$ & [23] & $55.6(52.6 ; 58.5)$ & $48.1(45.7 ; 50.4)$ \\
\hline ADL, $0-100^{*}$ & $1.6(-4.4 ; 7.6)$ & $2.8(-9.6 ; 15.3)$ & [23] & $71.7(68.2 ; 75.1)$ & $62.9(59.8 ; 66.0)$ \\
\hline Pain, 0-100 & $-12.2(-17.2 ;-7.1)$ & $-23.1(-35.4 ;-10.8)$ & {$[16]$} & $47.0(40.5 ; 53.5)$ & $55.7(52.0 ; 59.5)$ \\
\hline \multicolumn{6}{|l|}{ AUSCAN } \\
\hline Function, 0-36 & $-3.3(-5.7 ;-0.9)$ & $-9.8(-23.9 ; 4.2)$ & [23] & $17.1(15.3 ; 19.0)$ & $20.9(19.4 ; 22.3)$ \\
\hline Pain, 0-20 & $-1.1(-2.6 ; 0.4)$ & $-1.8(-18.5 ; 14.9)$ & [27] & $9.0(8.2 ; 9.9)$ & $11.1(10.3 ; 11.8)$ \\
\hline FIHOA, 0-30 & $0.1(-1.6 ; 1.7)$ & $22.7(-0.7 ; 46.0)$ & [23] & $12.4(11.1 ; 13.7)$ & $13.9(12.8 ; 15.0)$ \\
\hline VAS pain, 0-100 & $-11.5(-18.2 ;-4.7)$ & $-24.4(-36.1 ;-12.8)$ & [27] & $47.7(42.2 ; 53.3)$ & $48.8(44.6 ; 53.0)$ \\
\hline
\end{tabular}

Direction of effect of all instruments is higher is worse, except those with *.†For all MCll direction of effect was unified, so positive values indicate worse symptoms and negative values values indicate improved symptoms.

Conclusion: The only function instrument with an acceptable threshold for MCII was AUSCAN function, while for pain MHQ and VAS performed better than AUSCAN. PASS values show a relatively high level of tolerance of $50 \%$ of the maximum of the scale. Disclosure of Interests: Féline Kroon: None declared, Lotte van de Stadt Grant/ research support from: The HOSTAS and HOPE studies were sponsored by the Dutch Arthritis Society., Désirée van der Heijde: None declared, Margreet Kloppenburg Grant/research support from: The HOSTAS and HOPE studies were sponsored by the Dutch Arthritis Society.

DOI: 10.1136/annrheumdis-2021-eular.400

\section{POS0183 SIGLEC1 AS A TYPE I INTERFERON BIOMARKER IN IDIOPATHIC INFLAMMATORY MYOPATHIES}

M. Graf ${ }^{1}$, A. S. L. Von Stuckrad ${ }^{2}$, A. Uruha ${ }^{3,4}$, J. Klotsche ${ }^{5}$, L. Zorn-Pauly ${ }^{1}$ N. Unterwalder ${ }^{6}$, T. Buttgereit ${ }^{7}$, M. Krusche ${ }^{1}$, C. Meisel ${ }^{6}$, G. R. Burmester ${ }^{1}$, F. Hiepe ${ }^{1}$, R. Biesen ${ }^{1}$, T. Kallinich ${ }^{2}$, W. Stenzel ${ }^{4}$, U. Schneider ${ }^{1}$, T. Rose ${ }^{1}$. ${ }^{1}$ Charité - Universitätsmedizin Berlin, Rheumatology and Clinical Immunology, Berlin, Germany; ${ }^{2}$ Charité - Universitätsmedizin Berlin, Pediatric Pneumology, Immunology and Critical Care Medicine and SPZ (Center for Chronically Sick Children), Berlin, Germany; ${ }^{3}$ Tokyo Metropolitan Neurological Hospital, Neurology, Tokyo, Japan; ${ }^{4}$ Charité - Universitätsmedizin Berlin, Neuropathology, Berlin, Germany; ${ }^{5}$ Deutsches Rheuma-Forschungszentrum Berlin (DRFZ), DRFZ, Berlin, Germany; ${ }^{6}$ Labor Berlin - Charité Vivantes GmbH, Immunology, Berlin, Germany; ${ }^{7}$ Charité - Universitätsmedizin Berlin, Dermatology and Allergy, Dermatological Allergology, Berlin, Germany

Background: Idiopathic inflammatory myopathies (IIM) are autoimmune diseases that mainly affect skeletal muscle, lung, skin and joints. IIM can be separated into dermatomyositis (DM), inclusion body myositis (IBM), antisynthetase syndrome (AS) and immune-mediated necrotizing myopathy (IMNM). Type I interferons (IFN) are known to play a crucial role in the etiopathogenesis of some of these entities such as DM.[1] Sialic acid binding Ig-like lectin 1 (SIGLEC1, CD169) is part of the type I IFN signature found in SLE and DM and is expressed on the cell surface of monocytes. Thus, analysis of SIGLEC1 expression by flow cytometry enables a straightforward assessment of the type I IFN signature. Its utility has been shown for juvenile and adult SLE and other rheumatic diseases but not in IIM.[2,3] The assessment of the type I IFN system in clinical practice is an unmet need and, in this context, SIGLEC1 might be useful. Objectives: To assess SIGLEC1 expression on monocytes by flow cytometry as a type I IFN biomarker in IIM

Methods: Pediatric and adult patients with a clinical diagnosis of DM, AS, IMNM and IBM and at least one measurement of SIGLEC1 who have been treated at the Department of Rheumatology, Charite - Universitätsmedizin Berlin between 2015 and 2020 were included in this retrospective study. Control groups of healthy individuals $(n=19)$ and SLE patients $(n=30)$ were included. Disease activity was assessed by Physician Global Assessment (PGA) and Childhood Myositis Assessment Scale (CMAS). SIGLEC1 expression on monocytes was analyzed by flow cytometry. Cross-sectional analyses $(n=74)$ were performed using Mann Whitney-U test (MWU) and two-level mixed-effects linear regression model was used for longitudinal analyses ( $n=26,110$ visits). This study was approved by the local ethics committee of the Charité - Universitätsmedizin Berlin.

Results: 74 patients (adult/juvenile DM: $n=21 / n=17$; AS: $n=19$; IMNM: $n=8$; IBM: $n=9$ ) were included. In cross-sectional analysis, SIGLEC1 expression was significantly upregulated in adult and juvenile DM patients with moderate to severe disease activity $(P G A \geq 5)$ compared with adult/juvenile $D M$ patients with no to moderate disease activity $(P G A<5)$ (both $p<0.001)$. In longitudinal analyses, SIGLEC1 correlated with disease activity in juvenile DM (SIGLEC1 vs. CMAS: betaST $=-0.65 ; p<0.001$ ) and adult DM (SIGLEC1 vs. PGA: betaST $=0.52$; $p<0.001)$, better than Creatine Kinase $(C K)$ (juvenile DM, CK vs. CMAS: betaST $=-0.50 ; p<0.001$; adult $D M, C K$ vs $P G A$ : betaST $=0.17 ; p=0.149$ ). In AS $42,1 \%$ of the patients showed elevated SIGLEC1 expression, while it was not upregulated in IMNM and only in two patients with IBM, who were concurrently positive for autoantibodies that affect the type I IFN system (see Figure 1).
Conclusion: SIGLEC1 is a useful biomarker to identify an activated type I IFN system in IIM. Flow cytometry is used widely in laboratory medicine, which could facilitate the implementation of SIGLEC1 into clinical routine.

\section{REFERENCES:}

[1] Gallay L, Mouchiroud G, Chazaud B. Interferon-signature in idiopathic inflammatory myopathies: Current Opinion in Rheumatology 2019;31:63442. doi:10.1097/BOR.0000000000000653

[2] Rose $\mathrm{T}$, Grutzkau A, Hirseland $\mathrm{H}$, et al. IFNalpha and its response proteins, IP-10 and SIGLEC-1, are biomarkers of disease activity in systemic lupus erythematosus. Ann Rheum Dis 2013;72:1639-45. doi:10.1136/ annrheumdis-2012-201586

[3] Stuckrad SL von, Klotsche J, Biesen R, et al. SIGLEC1 (CD169) is a sensitive biomarker for the deterioration of the clinical course in childhood systemic lupus erythematosus. Lupus 2020;:961203320965699. doi:10.1177/0961203320965699



Figure 1. SIGLEC1 expression on monocytes in IIM subgroups and control groups; in IIM subgroups, patients with low disease activity $(P G A<5)$ are marked in blue, patients with high disease activity $(P G A \geq 5)$ are marked in red; $m A b / c e l l$, monoclonal antibodies bound per cell

Disclosure of Interests: None declared

DOI: 10.1136/annrheumdis-2021-eular.2816

\section{POS0184 URINE-GALECTINE 3 BINDING PROTEIN (U-GAL3BP) IS A SENSITIVE MARKER OF KIDNEY INFLAMMATION} AND RESPONSE TO TREATMENT IN LUPUS

F. Faustini ${ }^{1,2}$, H. Idborg ${ }^{2}$, E. Svenungsson ${ }^{1,2}$, S. Poetzsch ${ }^{3}$, S. Okitsu ${ }^{4}$, A. Larsson ${ }^{5}$, I. Gunnarsson ${ }^{1,2} .{ }^{1}$ Karolinska University Hospital, Rheumatology, Stockholm, Sweden; ${ }^{2}$ Karolinska Institutet, Department of Medicine, Division of Rheumatology, Stockholm, Sweden; ${ }^{3}$ Merck Healthcare KGaA, Merck Healthcare KGaA, Darmstadt, Germany, ${ }^{4} E M D$ Serono Research and Development Institute, EMD Serono Research and Development Institute, Billerica, MA, United States of America; ${ }^{5}$ Uppsala University, Department of Medical Sciences/Clinical Chemistry, Uppsala, Sweden 
Background: Lupus nephritis (LN) represents a serious manifestation of systemic lupus erythematosus (SLE) which requires timely diagnosis, treatment and monitoring. Kidney biopsy is the gold standard of diagnosis and is instrumental to treatment decisions, however it is not generally performed for monitoring and evaluation of response to treatment. To such purposes, accessible biomarkers, for instance urinary, might be highly advantageous.

Objectives: To evaluate urine-Galectin 3 binding protein (UGAL3BP) as a novel biomarker in biopsy-proven active lupus nephritis (A-LN) in comparison to active non-renal SLE (ANR-SLE), inactive SLE (I-SLE), and in population-based controls $(\mathrm{HC})$. Furthermore, we compared UGAL3BP with known markers of renal pathology including neutrophil gelatinase-associated lipocalin (NGAL), osteopontin (OPN), kidney injury molecule $1(\mathrm{KIM}-1)$, and galectin 3 (GAL3).

Methods: Urine samples from A-LN ( $n=86)$, ANR-SLE $(n=63)$, I-SLE $(n=73)$ and $\mathrm{HC}(\mathrm{n}=48)$ were included. UGAL3BP was measured using a commercial ELISA kit and values, adjusted for u-creatinine levels, were expressed as $\mathrm{ng} / \mathrm{mmol}$. Other markers analyzed according to clinical routine at the Department of Clinical Chemistry at Uppsala University Hospital were also adjusted for u-creatinine levels. Renal biopsies were graded according to the ISN/RPS classification(1) and evaluated for activity and chronicity index. Ten A-LN patients were evaluated before and after induction treatment.

Results: In the A-LN group, median (IQR) levels of u-GAL3BP were 15.8 (6.824.6) $\mathrm{ng} / \mathrm{mmol}$, while in ANR-SLE, I-SLE, HC were significantly lower 4.4 (2.09.0), $2.8(1.7-4.7), 2.0(0.9-4.8)$ respectively (Kruskal-Wallis $p<0.0001)$. Similarly, u-NGAL was found at higher levels in A-LN patients, $3.3(2.0-5.7) \mu \mathrm{g} / \mathrm{mmol}$, with respect to the ANR-SLE 2.0 (0.9-4.5), I-SLE 1.6 (0.8-3.2), and HC 2.4 (1.2-5.3), $(p=0.008)$. The highest levels of OPN were found in the group of I-SLE (190.6 (85.1-299.9) $\mathrm{\mu g} / \mathrm{mmol}$, compared to A-LN 72.98 (37.6-118.1), ANR-SLE 92.3 (58.5-129.7) and HC 76.5 (58.2-120.3), ( $<<0.0001)$. KIM-1 levels differed among groups with higher levels in the A-LN group (188.9 (113.7-309.7) $\mathrm{ng} / \mathrm{mmol})$, in comparison to ANR-SLE 131.4 (92.2-186.1), I-SLE 123.8 (70.3-200.2), and HC 78.2 (68.8-115.1), $(p<0.0001)$. GAL3 showed comparable levels across groups. When exploring the biomarkers across histologic subgroups of LN, U-GAL3BP could discriminate between proliferative and mesangial forms (17.7(9.6-32.5) vs $6.7(5.1-16.1) \mathrm{ng} / \mathrm{mmol}, \mathrm{p}=0.027)$, while it did not discriminate against membranous LN. U-NGAL was higher in proliferative LN 3.7(2.4-5.8) $\mu \mathrm{g} / \mathrm{mmol}$ with respect to membranous $2.4(1.1-3.8)(p=0.01)$, while mesangial LN showed comparable levels. OPN, KIM-1 and GAL3 were comparable across groups.

In the ten patients with available samples after induction therapy (mycophenolate mofetil (MMF) in 4, rituximab (RTX) in one, cyclophosphamide in 5 (one combined with MMF and one with RTX), u-GAL3BP showed a significant decrease of median levels from 218.8 to $41.5 \mathrm{ng} / \mathrm{mmol}$ (Wilcoxon $\mathrm{p}=0.03$ ). $\mathrm{u}$-GAL3BP associated with renal activity in class III/IV LN $(R=0.42, p=0.004)$.

Conclusion: Among the tested markers, high uGal3BP adjusted for creatinine was found to be a promising marker of renal involvement in SLE patients and associated with renal activity in patients with proliferative forms (class III/IV) of LN. A decrease was further seen following therapy, suggesting that $u$ GAL3-BP could be used to monitor renal activity.

REFERENCES:

[1] Weening JJ, D’Agati VD, Schwartz MM, Seshan SV, Alpers CE, Appel GB, et al. The classification of glomerulonephritis in systemic lupus erythematosus revisited. J Am Soc Nephrol. 2004;15(2):241-50.

[2] Houssiau FA, Vasconcelos C, D'Cruz D, Sebastiani GD, Garrido Ed Ede R, Danieli MG, et al. Immunosuppressive therapy in lupus nephritis: the Euro-Lupus Nephritis Trial, a randomized trial of low-dose versus high-dose intravenous cyclophosphamide. Arthritis Rheum. 2002;46(8):2121-31.

Disclosure of Interests: Francesca Faustini Speakers bureau: I have received speaking fees, last time more than two years ago, Helena Idborg: None declared, Elisabet Svenungsson: None declared, Sven Poetzsch Employee of: Merck Serono, Shinji Okitsu Employee of: Merck Serono, Anders Larsson: None declared, Iva Gunnarsson: None declared

DOI: 10.1136/annrheumdis-2021-eular.3477

\section{POS0185 IDENTIFICATION AND VALIDATION OF TWO NOVEL SERUM BIOMARKERS ASSOCIATED WITH THE SEROLOGICAL STATUS OF RHEUMATOID ARTHRITIS PATIENTS.}

V. Calamia ${ }^{1}$, L. Lourido ${ }^{1}$, P. Fernández Puente ${ }^{1,2}$, A. Illiano ${ }^{3}$, R. Paz González ${ }^{1}$, B. Rocha Loureda ${ }^{1}$, L. Collado Rodríguez ${ }^{1}$, E. Perez-Pampín ${ }^{4}$, C. RuizRomero ${ }^{1}$, A. Gonzalez ${ }^{4}$, F. J. Blanco' ${ }^{1}{ }^{1}$ Instituto de Investigación Biomédica de A Coruña (INIBIC), Complexo Hospitalario Universitario de A Coruña (CHUAC), Sergas, Universidade da Coruña (UDC), Unidad de Proteómica, Grupo de Investigación de Reumatología (GIR), A Coruña, Spain; ${ }^{2}$ Universidad de A Coruña (UDC), Centro de Investigaciones Científicas Avanzadas (CICA), A Coruña, Spain; ${ }^{3}$ University of Naples Federico II, Department of Chemical Sciences, Naples, Italy; ${ }^{4}$ Instituto de Investigación Sanitaria (IDIS). Hospital Clínico Universitario de Santiago (CHUS), Experimental and Observational Rheumatology and Rheumatology Unit, Santiago de Compostela, Spain
Background: Despite the diagnostic value of Rheumatoid Factor (RF) and Anti-Citrullinated Protein Antibodies (ACPA), more serological markers are needed in order to improve early diagnosis and treatment response of the Rheumatoid Arthritis (RA) patients. Increased knowledge about how these two major autoreactivities arise is crucial for understanding how RA develops and what mechanisms drive pathogenesis.

Objectives: We aimed to investigate, using a proteomic strategy, novel protein biomarkers associated with RF and/or ACPA that might be useful to stratify seropositive and seronegative RA patients.

Methods: A shotgun proteomic analysis was performed on 80 sera from the RA cohort of the Rheumatology Unit of the University Hospital of Santiago de Compostela (CHUS). Sera were classified as seropositive or seronegative according to their RF and ACPA values, and were then analyzed employing the iTRAQ labelling technique (Sciex) followed by LC-MALDI-MS/MS analysis (MALDI-TOF). A Multiple Reaction Monitoring (MRM) method was subsequently developed using the Skyline Software for the simultaneous quantification of 26 peptides belonging to ten putative protein biomarkers. The quantitative targeted analysis was performed using peptides with isotope labelled amino acids as internal standards Serum levels of orosomucoid 1 (ORM1) and haptoglobin (HPT) were measured using commercially available ELISA Kits in the whole RA cohort $(n=260)$ from the Rheumatology Unit of the University Hospital of A Coruña (HUAC).

Results: For the initial screening, eighty sera were grouped according to the ACPA RF status in 4 pools (20 patients/pool). Using an iTRAQ technology-based quantitative proteomic approach, the abundance of eleven proteins was altered in the sera from $A C P A^{\text {pos }} / R^{\text {pos }}, 13$ proteins in $A C P A^{\text {neg }} / R^{\text {pos }}$ and 12 proteins in ACPApos/RF ${ }^{\text {neg }}$, compared to $A C P A^{\text {neg }} / R^{\text {neg }}$. Vitamin $D$ binding protein (VTDB) was the unique protein that resulted increased in all the comparisons. For the biomarker verification phase, all the samples from the CHUS cohort were analyzed individually $(n=80)$. Using the MRM technology, 26 peptides belonging to ten putative protein biomarkers associated with double positivity were simultaneously quantified. The statistical analysis showed a significant modulation of 9 peptides (belonging to 4 different proteins) in $\mathrm{ACPA}^{\mathrm{pos}} / \mathrm{RF}^{\text {pos }}, 7$ peptides (5 proteins) in $\mathrm{ACPA}^{\text {neg }} / \mathrm{RF}^{\mathrm{pos}}$, and 9 peptides (6 proteins) in $A C P A^{\text {pos }} / R^{\text {neg }}$ compared to $A C P A^{\text {neg }} / R^{\text {neg }}(p<0.05)$. Two acute phase reactants (ORM1 and HPT) displayed the same modulation in both screening and verification phases, thus confirming their association with the double positivity. Finally, in the biomarker validation phase, a total of 260 patients from CHUAC were included (Table 1). RA patients were classified as follows: (1) 112 patients $(43.1 \%)$ were $\mathrm{ACPA}^{\mathrm{pos}} / \mathrm{RF}^{\mathrm{pos}}$; (2) 73 patients $(28.1 \%)$ were $\mathrm{ACPA}^{\text {neg }} / \mathrm{RF}^{\text {neg }}$ (3) 51 patients (19.6\%) were $\mathrm{ACPA}^{\text {neg }} / \mathrm{RF}^{\text {pos }}$; and (4) 24 patients $(9.2 \%)$ were ACPA${ }^{\mathrm{pos}} / \mathrm{RF}^{\mathrm{neg}}$. Serum levels of ORM1 and HPT (Figure 1), measured by commercial immunoassays, confirmed their increased values in double seropositive patients $(p=0,0053$ ORM1; $p=0,0026$ HPT). Finally, the increased level of ORM1 resulted associated with RF rather than ACPA status $\left(p=0,0008 A C P A^{\text {neg }} / R^{p 0 s}\right)$; whereas HPT was associated with ACPA rather than $R F$ status $\left(p=0,0112 A^{A C P A}{ }^{p o s} / R F^{\text {neg }}\right.$ ).

Table 1. The different phases of RA biomarker development followed in this study.

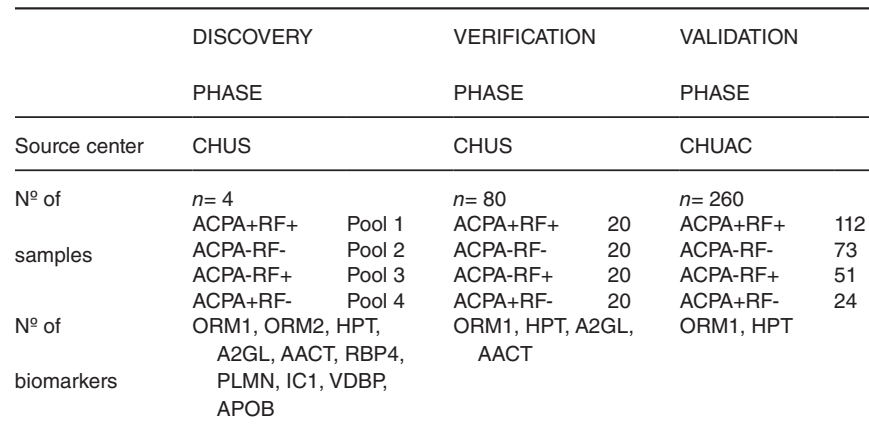
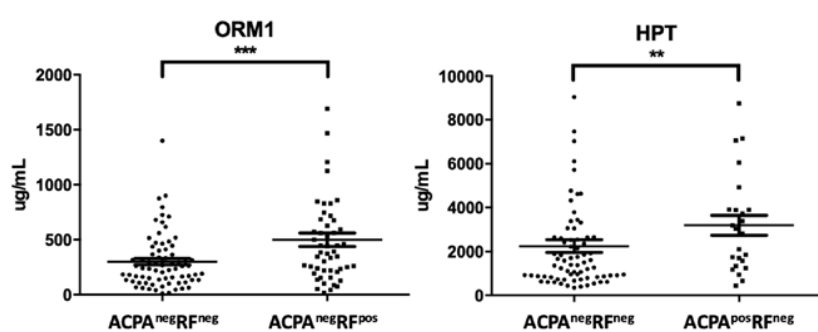

Figure 1: ORM1 levels are increased in RA patients seropositive for RF $(499 \pm 437 \mathrm{ug} / \mathrm{mL}$ in $A_{C P A^{\text {neg }}} R^{\text {pos }}$ vs $300 \pm 248 \mathrm{ug} / \mathrm{mL}^{\text {in }}$ ACPA $\left.{ }^{\text {reg RF }} \mathrm{F}^{\text {neg }}\right)$. HPT levels are statistically significant increase in RA patients seropositive for ACPA (3193+2233 ug/mL in ACPA ${ }^{\text {pos RF }}{ }^{\text {neg }} \mathrm{vs} 2046 \pm 1795 \mathrm{ug} / \mathrm{mL}$ in ACPA $^{\text {neg RF }}{ }^{\text {neg }}$.

Figure 1. 